Bundesinnenminister Dr. Hans-Peter Friedrich: „De-Mail ist eine leicht zu nutzende Technologie, mit der man im Internet sicher, vertraulich und verlässlich Nachrichten austauschen kann. Das gilt sowohl zwischen Privaten als auch für die Kommunikation mit Gerichten und Behörden."

Durch die Zusammenarbeit im E-Justice-Bereich wird der elektronische Zugang zu den Gerichten grundlegend modernisiert. Außerdem soll die Justiz künftig Urteile, Beschlüsse, Schriftsätze und Ladungen rechtssicher und deutlich kostengünstiger elektronisch zustellen können.

Begleitende Änderungen der Verfahrensordnungen, die erforderlich sind, um die Zugangshürden für eine elektronische Kommunikation mit der Justiz bedeutend zu senken, werden derzeit durch das Bundesministerium der Justiz geprüft.

Darüber hinaus erarbeitet das Bundesministerium des Innern derzeit den Entwurf für ein E-Government-Gesetz des Bundes mit dem Ziel, für die Bürgerinnen und Bürgern sowie für die Wirtschaft die elektronische Kommunikation mit der Verwaltung zu erleichtern. Auch hierbei wird De-Mail neben der eID-Funktion des neuen Personalausweises eine wichtige Rolle spielen.

\section{Bund übernimmt Vorsitz des IT-Planungsrats}

Am 1. Januar 2012 hat die IT-Beauftragte der Bundesregierung, Staatssekretärin Cornelia Rogall-Grothe, planmäßig für ein Jahr den Vorsitz im IT-Planungsrat übernommen. Der IT-Planungsrat ist das oberste Gremium von Bund und Ländern für die Steuerung der Informationstechnik des Staates.

2011 hat der IT-Planungsrat unter dem Vorsitz des Landes Baden-Württemberg u. a. die Umsetzung der nationalen E-Government Strategie in deutschen Behörden auf den Weg gebracht. Aufgebaut wird dabei auf bereits laufenden Projekten wie dem neuen Personalausweis, der auch im Internet Sicherheit bietet, der einheitlichen Behördennummer 115 und "De-Mail“.

Der scheidende Vorsitzende des IT-Planungsrats, Ministerialdirektor Dr. Herbert Zinell, Amtschef des Innenministeriums BadenWürttemberg, zieht zum Jahresende eine positive Bilanz: „Im Jahr 2011 hat der IT-Planungsrat maßgebliche Projekte zur Umsetzung der Nationalen E-Government-Strategie begonnen. Sie runden die bereits bundesweit laufenden IT-Maßnahmen ab und führen darüber hinaus. Dadurch können alle Behörden, aber auch die Städte, Gemeinden und Kreise, ihre IT-Nutzung sicherer und wirtschaftlicher gestalten."

Die IT-Beauftragte der Bundesregierung will diese Arbeiten konsequent fortführen. „Für die Aufgabenerfüllung des Staates ist der Einsatz der Informationstechnik von zentraler Bedeutung. Bund und Länder werden unter Steuerung durch den IT-Planungsrat die verschiedenen IT-Systeme zu einer föderalen IT-Infrastruktur zusammenführen. Wichtige Entscheidungen zur IT-Harmonisierung sind bereits getroffen. Unter dem Vorsitz des Bundes werden wir in 2012 im IT-Planungsrat an weiteren Schritten zur Gemeinsamkeit arbeiten", so Rogall-Grothe.

Ein Schwerpunkt ist die IT-Sicherheit. Der IT-Planungsrat hat im Oktober 2011 ein Konzept für eine Leitlinie Informationssicherheit verabschiedet, die auch verbindliche IT-Sicherheitsstandards enthält. Ministerialdirektor Dr. Zinell: „Die steigende Anzahl an Sicherheitsvorfällen macht das Arbeiten im Verbund immer bedeutsamer. Die Errichtung eines gemeinsamen Warn- und Informations- dienstes, an dem wir intensiv arbeiten, ist eine wichtige Maßnahme."

Um den europäischen Austausch zu befördern, wird der IT-Planungsrat im kommenden Jahr unter anderem in Brüssel tagen. Hierzu erklärt Staatssekretärin Rogall-Grothe: „Die Zusammenarbeit in Europa wird auch in der IT immer wichtiger. Wir sind bereits jetzt vielfach grenzüberschreitend vernetzt. Dies muss sich auch durch eine angemessene Koordinierung und Harmonisierung unserer IT-Landschaften nicht nur über föderale, sondern - in angemessenem Umfang - auch über nationale Grenzen hinweg ausdrücken."

Die nächste Sitzung des IT-Planungsrats findet am 8. März 2012 auf der CeBIT in Hannover statt.

\section{Studie: Selbst- und Fremdbild von Datenschutzbeauftragten}

Außenstehende schätzen Datenschutzbeauftragte überwiegend als "wichtig" oder "eher wichtig" ein. Gleichzeitig nehmen sie diese als "Papiertiger" wahr, die in Organisationen und Unternehmen einen relativ geringen Einfluss haben. Dies ist eins der Ergebnisse der am 08.12.2011 veröffentlichten Studie der Universität OIdenburg und des Berufsverbands der Datenschutzbeauftragten Deutschlands (BvD) zum „Selbst- und Fremdbild von Datenschutzbeauftragten". Autorlnnen sind Dr. Herbert Schulze und Dipl.Wi.Jur.(FH) \& MA Lena Marie Glunz vom Institut für Betriebswirtschaftslehre und Wirtschaftspädagogik der Universität Oldenburg.

Der NRW-Landesdatenschutzbeauftragte Ulrich Lepper, der die Studie als einen weiteren wichtigen Baustein des BvD für die Qualität der Datenschutzberatung sieht, wertet das Ergebnis der Studie „als eindeutigen Hinweis, dass qualifizierte Datenschutzbeauftragte ein Muss sind".

Die explorative Studie mit über $6.000 \mathrm{im}$ Frühjahr befragten Teilnehmerlnnen macht deutliche Diskrepanzen zwischen der Eigeneinschätzung und Außenwahrnehmung aus. Sie dokumentiert zudem, dass sowohl über 40 Prozent der Nicht-Datenschutzbeauftragten als auch der überwiegende Teil der Datenschutzbeauftragten die Qualifikation als zu gering einordnet. Vor allem Berufsfremde erwarten eine vollständige Berufsausbildung oder gar ein Aufbaustudium für Datenschutzbeauftragte.

Thomas Spaeing, Vorstandsvorsitzender des BvD, bewertet diese Ergebnisse als „schallende Ohrfeige" für die oft angebotenen dreitägigen Ausbildungskurse zum Datenschutzbeauftragten. „Die Realität der Ausbildung liegt weit entfernt von den Erwartungen - und den tatsächlichen Anforderungen", betont Spaeing. Der Berufsverband sehe sich durch die Oldenburger Studie in seiner Arbeit bestätigt, für erheblich mehr Qualität bei der Qualifikation von Datenschutzbeauftragten zu sorgen.

Die Studie zeigt Tendenzen auf, dass die betrieblichen und behördlichen Datenschutzbeauftragten ihre Kernkompetenzen in der Beratung sehen, während die Nicht-Datenschutzbeauftragten mit der Funktion vor allem Überprüfung und Kontrolle verbinden. „Wir müssen uns selbstkritisch fragen, ob wir Datenschutzbeauftragten unsere Aufgaben ausreichend kennen, erklärt der stellvertretende BvD-Verbandsvorsitzende Marco Biewald. Die Studie mache einmal mehr den Bedarf nach einem verbindlichen, eindeutigen Berufsbild deutlich. 